\title{
Influencia de los programas de planificación familiar en la mortalidad perinatal
}

\author{
Moisés Huamán*
}

\section{Introducción}

América Latina en el año 95 tenía una población mayor de 470 millones de habitantes, con un mosaico de diversas y contrastantes realidades, tanto como países como regiones; es así por ejemplo la gran diferencia del ingreso percápita anual. En la década del 80, "Década económicamente perdida", se afectó más la economía sobre todo por causas externas, desastres naturales, luchas por el poder y las diferentes formas de concebir la organización social, adicionándose en algunos países la violencia y la inestabilidad política que obliga a los gobiernos a reorientar los escasos recursos económicos; esto ha llevado a un aumento del porcentaje de hogares en situación de pobreza (del 35\% el año 80 al 39\% el año 90) y de pobreza crítica (15\%el año 80 al $18 \%$ el año 90$)$, lo cual no sólo se debió al crecimiento poblacional. Al final del siglo no se espera mayores mejoras de esta situación, debido a que el auge económico de algunos países no ha mejorado la situación de pobreza de las grandes mayorías, por la falta de políticas con justicia distributiva (SEPAL) (8).

Los promedios de los indicadores nacionales en salud no reflejan la real situación, encubriendo grandes desigualdades internas que se polarizan más en las zonas rurales y en los barrios marginales. La urbanización de la economía obligó a partir de la época del 50 a grandes corrientes migratorias hacia las ciudades, es así que en América Latina se encuentran las ciudades más populosas del mundo, y entre Ciudad de México, Gran Buenos Aires y Sao Paulo suman el $10 \%$ de la población de América Latina.

Actualmente, la población rural en América Latina es menos de la tercera parte de la población total, con puntuales diferencias en países como Guatemala, Honduras, El Salvador y Paraguay, que tienen mayor porcentaje de población rural.

Por otro lado, la maternidad-fecundidad son conceptos culturales con diferente valor en las diferentes estructuras sociales, influidas por su propia historia cultural, el papel de la mujer en ese contexto social, creencias religiosas, etc.

Es necesario considerar la importancia de las características sociodemográficas, económicas y culturales y en

Sociedad Peruana de Obstetricia y Ginecología. Doctor en Medicina. Av. Aramburú No. 231 Of. 3 - Lima 27 - Perú. las diferentes áreas poblacionales, en el avance de los programas preventivos poblacionales y la disminución de los indicadores negativos de salud.

\section{Salud perinatal}

La Salud perinatal es un proceso dinámico resultante de la capacidad de la madre y del producto de la gestación para interactuar satisfactoriamente entre sí y con el medio ambiente externo.

Los factores asociados en la salud perinatal, tienen que ver con la situación de la salud integral de la madre desde la etapa preconcepcional, factores socioculturales, económicos y ambientales; y la existencia, calidad y nivel de utilización de los servicios de salud obstétricos y neonatales. Estos factores condicionarán el menor o mayor riesgo para la muerte perinatal, dado que influyen en el crecimiento y la nutrición fetal, riesgos para malformaciones, infecciones perinatales, asfixia, parto prematuro, etc.

En la mortalidad fetal, la mitad de casos son de causa desconocida, siendo el origen placentario el $60 \%$ de las causas desconocidas, siguiendo las malformaciones congénitas entre el 20 al $30 \%$ de causas.

La mortalidad neonatal, se asocia fundamentalmente al bajo peso al nacer, la prematuridad y la asfixia perinatal. Son factores de riesgo del bajo peso al nacer: la multiparidad, malnutrición materna, hipertensión crónica, mala historia obstétrica, embarazos múltiples, poca ganancia ponderal, factores genéticos maternos, sangrados placentarios, anemia, RPM, anomalías fetales, edades extremas de la vida reproductiva de la mujer, bajo nivel socioeconómico y cultural, tabaco, drogas, residencia en grandes alturas, inadecuado control prenatal, unión inestable de la pareja, entre otros.

La influencia de la situación económica en la mortalidad perinatal (MPN) se evidencia en el incremento de ésta en los períodos de crisis económica de los países en vías de desarrollo, como lo demuestra un estudio en el área urbano-marginal de Lima donde se triplicó la MPN durante la crisis económica de la década pasada.

$\mathrm{Al}$ evaluar las causas, los factores asociados y los factores de riesgo para las entidades más importantes en la MPN, se observa que muchos de ellos tienen estrecha relación con el crecimiento demográfico, por tanto, con el avance de los programas de salud reproductiva y planificación familiar. Es así que los Indicadores Específicos para evaluar los programas para la reducción de la 
MPN, se refiere a planificación familiar, nutrición, control prenatal y el parto.

\section{Planificación familiar}

América Latina se ubica entre las regiones con una población joven, con altas tasas de fecundidad, calculándose para el quinquenio 95-2000 una tasa global de fecundidad (TGF) de 2.8, la cual esconde sustanciales diferencias entre los países y dentro de ellos las regiones más desprotegidas; es así que Guatemala, Haití, Nicaragua, Honduras, Paraguay y Bolivia tienen TGF mayores de 4 y Cuba, Chile, Jamaica, Martinica, Uruguay, Puerto Rico, Argentina y otros tienen TGF menores de 3.

El Perú tiene una alta TGF, con un crecimiento poblacional de $2 \%$ anual, con malos indicadores en salud materna infantil (Tasa de Mortalidad Materna de alrededor de 300 por 100 mil nacidos vivos y tasa de mortalidad infantil de 55 por 1000 nacidos vivos). Estos indicadores sociodemográficos, comparados con el de los países desarrollados, demuestran claramente la relación estrecha ante el exagerado crecimiento poblacional, las malas condiciones de salud y las altas tasas de muerte en las poblaciones más desprotegidas y en las personas más vulnerables como son las gestantes y los productos de la concepción hasta la etapa neonatal.

Se observa relación directa entre la Tasa Global de Fecundidad y la Tasa de Mortalidad Infantil (Cuadros 1 y 2).

Cuadro 1

AMERICA LATINA: TASA GLOBAL DE FECUNDIDAD SEGUN ALGUNOS PAISES, 1995-2000*

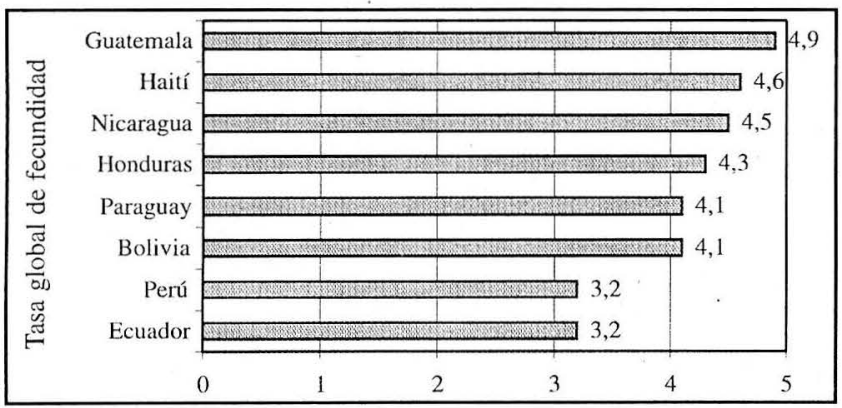

Cuadro 2

AMERICA LATINA: TASA DE MORTALIDAD INFANTIL, SEGUN ALGUNOS PAISES, 1990-1995*

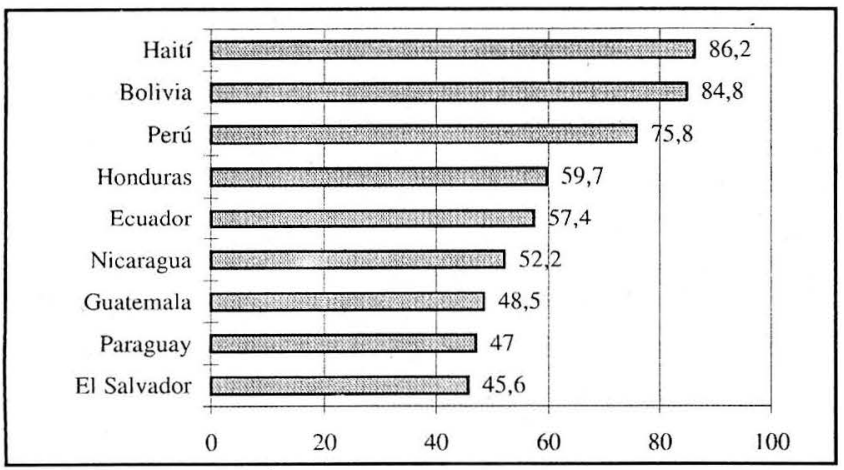

* Instituto Nacional de Estadística e Informática, Perú (8).

\section{Mortalidad perinatal}

En la mayoría de los países de América Latina no se cuenta con tasas de mortalidad perinatal exactas, existe un importante subregistro, reportándose con mayor frecuencia tasas hospitalarias, las que tienen un amplio rango de variación (entre 18 a 40 por mil n.v.), siendo las causas similares para grupos de peso adecuado, bajo peso y muy bajo peso al nacer. '

La MPN es más frecuente en gestantes de alto riesgo, en un estudio nuestro en más del $90 \%$, asociándose básicamente a madres de los extremos de la vida reproductiva (menores de 16 años o mayores de 35 años), período internatal corto, primiparidad, enfermedades crónicas como diabetes, cariopatías, nefropatías, etc., desnutrición materna, gran multiparidad; muchos de estos factores modificables con un adecuado espaciamiento de los embarazos, sobre todos los factores de riesgo relacionados a la edad de la gestante, multiparidad y período internatal corto, como lo demuestra el siguiente estudio:

\section{EFECTO DEL ESPACIAMIENTO DE LOS NACIMIENTOS} EN LA MORTALIDAD INFANTIL EN EL PERU

\begin{tabular}{|cc|}
\hline $\begin{array}{c}\text { Período internatal } \\
\text { (meses) }\end{array}$ & $\begin{array}{c}\text { Mortalidad infantil } \\
\text { (por mil n.v.) }\end{array}$ \\
\hline$<24$ & 137.1 \\
$24-47$ & 86.6 \\
$>48$ & 54.9 \\
\hline
\end{tabular}

* Fuente: Encuesta Mundial de Fecundidad (1977-1978).

Diferentes estudios demuestran que el período internatal menor de 2 años duplica el riesgo de mortalidad infantil que los que nacen después de $\operatorname{los} 2$ años (Cuadros 3 y 4 ).

El riesgo de un infante de morir, se duplica cuando es el cuarto o el quinto hijo en relación al segundo y tercero. Igualmente se ha demostrado que el infante cuyo hermano sucesivo nació en un período de 6 años, tiene el 50\% más de posibilidades de morir que si hubiese nacido después de los 2 años (1).

\section{Cuadro 3} PORCENTAJE DE NIÑOS NACIDOS CON PERIODO
INTERNATAL CORTO**

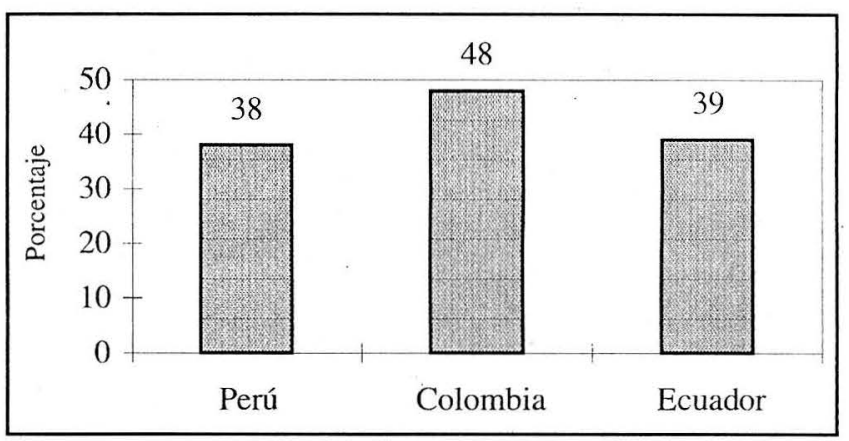


Cuadro 4

REDUCCION ESTIMADA DE MUERTES INFANTILES AL NACER CON INTERVALO MAYOR DE DOS AÑOS**

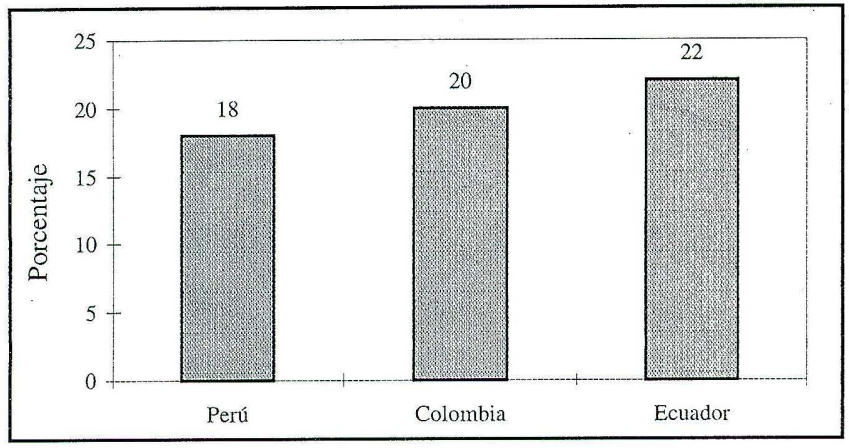

** Maine, 1987.

Hay consenso en reconocer que las principales estrategias para reducir la MPN son:

- Promoción de la salud integral de la mujer en toda su vida.

- Educación en salud reproductiva, sexualidad y planificación familiar, para evitar embarazos no deseados.

- Educación en salud a los futuros padres, informando las medidas de promoción y prevención de la salud infantil.

- Provisión de servicios de calidad para la atención de las mujeres en edad fértil y neonatos.

Es claro que la planificación familiar será un soporte importante en el desarrollo de estas estrategias.

La planificación familiar es una estrategia básica en la prevención de la mortalidad materna y perinatal con impacto a corto plazo, lo que permitiría programar y ejecutar otras estrategias a mediano y largo plazo.
En el Perú la mortalidad infantil para 1993 era de 58 por mil n.v., ésta encierra grandes diferencias entre las regiones de la costa, sierra y selva, entre el área rural y urbana, entre la capital del país y otras ciudades, etc. Es evidente la relación entre la tasa de mortalidad infantil y la tasa global de fecundidad, como pudo observarse en algunas regiones del país:

TASA GLOBAL DE FECUNDIDAD Y MORTALIDAD INFANTIL PERU, 1993

\begin{tabular}{|lcc|}
\hline & TGF & TMI \\
\hline Perú & 3.4 & 58.3 \\
Lima & 2.2 & 24.0 \\
Lampa (Sierra) & 4.7 & 103.3 \\
Loreto (Selva) & 7.4 & 80.4 \\
\hline
\end{tabular}

* Fuente: Censo de Población y Vivienda 1993.

En los últimos años en el Perú ha disminuido la mortalidad en menores de 5 años, debido a la disminución de las muertes por enfermedades inmunoprevenibles, enfermedad diarréica e infección respiratoria aguda, sin embargo se ha mantenido, aumentando proporcionalmente las muertes por causas asociadas al período perinatal y la desnutrición severa. Así en Lima el 37\% de la mortalidad infantil es por causas originadas en el período perinatal, siendo de $27 \%$ en el interior del país (8).

El avance lento de los programas de planificación familiar en la mayoría de los países latinoamericanos, determina que continuemos con altas tasas de mortalidad infantil y sobre todo mortalidad perinatal, al no reducirse los factores asociados a estas muertes, los que serían fácilmente modificables al cumplirse los objetivos de los programas de salud reproductiva y planificación familiar.

\section{BIBLIOGRAFIA}

1. Denegri J. Mortalidad Perinatal. Red Peruana de Perinatología. Lima, 1990; 21-26.

2. De la Cruz J. Mortalidad Perinatal en el período de 5 años 1985-1989 en la sección de Ginecología y Obstetricia del Hospital Militar C. Rev. Colombiana de Obstetricia y Ginecología 1991; 42(1): 37-48.

3. Freundl-G. Arch-Gynecol-Obstet. Developing-Countries; FamilyPlanning/*SN (Statics y Numerical data); Female; Germany/Ep. (Epidemiology); Human; Infant-NewBorn; Pregnancy; Reproducibility-of-Results. Vol 257 (1-4), p. 525-532: ISSN: 0937006711 Refs.

4. Gilman BI, Haustein D, Baldizon S. Rev. Médica de Supervivencia Infantil. En: Planificación Familiar: Un Programa que Salva Vidas No. 6 Mayo, 1989; 4-8.

5. Kilpatrick S-I, Jack Son-R, Croughan-Minihane M-S. Perinatal Mortality in Twins and Singletons matched for gestational age at delivery at $>$ or $=30$ weeks. USA. 1996 Jan., Vol. $174(1 p+1)$, p. 6671, ISSN: 0002-9378.
6. Klerman L-V, Phelan S-T, Poole, Goldenberg R-L. Family planning: an essential Component of prenatal care. In University of Alabama at Birmingham, USA. 1995 sept-oct, Vol 50 (5): P. 147-151, ISSN: 0098-8421 31 Refs.

7. Louw H-H, Khan M-B, Woods D-L, Power-M, Thompson M-C. Perinatal Mortality in the Cape Province, 1995 May, Vol. 85(5): p. 352-355, ISSN: 0038-2469.

8. Murillo F. Compendio de Estadísticas Sociales 1994-1995. Lima, septiembre de 1995.

9. Pinnoti, Aristodemo I, Faundes, A. Planificación Familiar y Mortalidad Perinatal (1996). Ginecología y Obstetricia. Brasil 1984; 7(1): 17-27.

10. Rojas AM. Mortalidad Perinatal y Materna. Rev. Colombiana de Obstetricia y Ginecología. Colombia 1990; 41(1): 33-38.

11. Torres Ruvalcava AB, Ramírez Andrade F, Azuara Bustamante J Torres Montes A, García Reyes J et al. Mortalidad Perinatal en San Luis Potosí. Boletín Médico del Hospital Infantil de México. 1990; 47(8): 543-550. 